\title{
A dynamical and radiation semi-analytical model of pulsar-star colliding winds along the orbit: Application to LS 5039
}

\author{
E. Molina and V. Bosch-Ramon
}

\begin{abstract}
Departament de Física Quàntica i Astrofísica, Institut de Ciències del Cosmos (ICCUB), Universitat de Barcelona (IEEC-UB), Martí i Franquès 1, 08028 Barcelona, Spain

e-mail: emolina@fqa.ub.edu, vbosch@fqa.ub.edu
\end{abstract}

Received 14 May 2020 / Accepted 30 June 2020

\begin{abstract}
Context. Gamma-ray binaries are systems that emit nonthermal radiation peaking at energies above $1 \mathrm{MeV}$. One proposed scenario to explain their emission consists of a pulsar orbiting a massive star, with particle acceleration taking place in shocks produced by the interaction of the stellar and pulsar winds.

Aims. We develop a semi-analytical model of the nonthermal emission of the colliding-wind structure, which includes the dynamical effects of orbital motion. We apply the model to a general case and to LS 5039.

Methods. The model consists of a one-dimensional emitter, the geometry of which is affected by Coriolis forces owing to orbital motion. Two particle accelerators are considered: one at the two-wind standoff location and the other one at the turnover produced by the Coriolis force. Synchrotron and inverse Compton emission is studied taking into account Doppler boosting and absorption processes associated to the massive star.

Results. If both accelerators are provided with the same energy budget, most of the radiation comes from the region of the Coriolis turnover and beyond, up to a few orbital separations from the binary system. Significant orbital changes of the nonthermal emission are predicted in all energy bands. The model allows us to reproduce some of the LS 5039 emission features, but not all of them. In particular, the $\mathrm{MeV}$ radiation is probably too high to be explained by our model alone, the $\mathrm{GeV}$ flux is recovered but not its modulation, and the radio emission beyond the Coriolis turnover is too low. The predicted system inclination is consistent with the presence of a pulsar in the binary.

Conclusions. The model is quite successful in reproducing the overall nonthermal behavior of LS 5039. Some improvements are suggested to better explain the phenomenology observed in this source, such as accounting for particle reacceleration beyond the Coriolis turnover, unshocked pulsar wind emission, and the three-dimensional extension of the emitter.
\end{abstract}

Key words. gamma rays: stars - radiation mechanisms: non-thermal - stars: winds, outflows - stars: individual: LS 5039

\section{Introduction}

Gamma-ray binaries are binary systems consisting of a compact object, which can be a black hole or a neutron star, and a nondegenerate star. These sources emit nonthermal radiation from radio up to very high-energy (VHE) gamma rays (above $100 \mathrm{GeV}$ ), and the most powerful ones host massive stars (see Dubus 2013; Paredes \& Bordas 2019, for a review). The main difference with X-ray binaries, which may also show persistent or flaring gamma-ray activity (see, e.g., Zanin et al. 2016; Zdziarski et al. 2018, for Cygnus X-1 and Cygnus X-3, respectively, and references therein), is that in X-ray binaries the (nonstellar) emission reaches its maximum at $\mathrm{X}$-rays, whereas gamma-ray binaries emit most of their radiation at energies above $1 \mathrm{MeV}$.

Thus far, there are nine confirmed high-mass gamma-ray binaries for which emission above $100 \mathrm{MeV}$ has been detected: LS I +61 303 (Tavani et al. 1998), LS 5039 (Paredes et al. 2000), PSR B1259-63 (Aharonian et al. 2005), HESS J0632+057 (Aharonian et al. 2007; Hinton et al. 2009), 1FGL J1018.65856 (Fermi LAT Collaboration 2012), HESS J1832-093 (Hess \& Abramowski 2015; Eger et al. 2016), LMC P3 (Corbet et al. 2016), PSR J2032+4127 (Abeysekara et al. 2018), and 4FGL J1405-6119 (Corbet et al. 2019). There are other candidate systems with a pulsar orbiting a massive star that exhibit nonthermal radio emission, but for which gamma rays have yet to be detected (see Dubus et al. 2017, and references therein).

The two most common scenarios proposed to explain the observed nonthermal emission in gamma-ray binaries involve either a microquasar, which generates nonthermal particles in relativistic jets powered by accretion onto a compact object (see, e.g., Bosch-Ramon \& Khangulyan 2009, for a thorough study of the scenario), or a nonaccreting pulsar, in which energetic particles are accelerated through shocks produced by the interaction of the stellar and pulsar winds (e.g., Maraschi \& Treves 1981; Leahy 2004; Dubus 2006; Khangulyan et al. 2007; SierpowskaBartosik \& Torres 2007; Zabalza et al. 2013; Takata et al. 2014; Dubus et al. 2015). With the contribution of orbital motion at large scales, this wind interaction leads to the formation of a spiral-like structure that is mainly composed of shocked pulsar material that can extend up to several dozens of times the orbital separation (see, e.g., Bosch-Ramon \& Barkov 2011; BoschRamon et al. 2012, 2015; Barkov \& Bosch-Ramon 2016, for an analytical description and numerical simulations of this structure).

In this work, we present a model to describe the broadband nonthermal emission observed in gamma-ray binaries through the interaction of the stellar and pulsar winds. The novelty of this model, with respect to previous works, is the application of 
Table 1. Parameters that are used in this work.

\begin{tabular}{lcc}
\hline \hline & Parameter & Value \\
\hline \multirow{2}{*}{ Star } & Temperature $T_{\star}$ & $4 \times 10^{4} \mathrm{~K}$ \\
& Luminosity $L_{\star}$ & $10^{39} \mathrm{erg} \mathrm{s}^{-1}$ \\
& Mass-loss rate $\dot{M}_{\mathrm{w}}$ & $3 \times 10^{-7} M_{\odot} \mathrm{yr}^{-1}$ \\
& Wind speed $v_{\mathrm{w}}$ & $3 \times 10^{8} \mathrm{~cm} \mathrm{~s}^{-1}$ \\
\hline Pulsar & Luminosity $L_{\mathrm{p}}$ & $3 \times 10^{36} \mathrm{erg} \mathrm{s}^{-1}$ \\
& Wind Lorentz factor $\Gamma_{\mathrm{p}}$ & $10^{5}$ \\
\hline \multirow{3}{*}{ System } & Orbital separation $D$ & $3 \times 10^{12} \mathrm{~cm}$ \\
& Orbital period $T$ & 5 days \\
& Orbital eccentricity $e$ & 0 \\
& Distance to the observer $d$ & $3 \mathrm{kpc}$ \\
& Nonthermal fraction $\eta_{\mathrm{NT}}$ & 0.1 \\
& Acceleration efficiency $\eta_{\mathrm{acc}}$ & 0.1 \\
& Injection power-law index $p$ & -2 \\
& Coriolis turnover speed $v_{\mathrm{Cor}}$ & $3 \times 10^{9}, 10^{10} \mathrm{~cm} \mathrm{~s}^{-1}$ \\
& Magnetic fraction $\eta_{B}$ & $10^{-3}, 10^{-1}$ \\
& System inclination $i$ & $30^{\circ}, 60^{\circ}$ \\
\hline
\end{tabular}

Notes. The last three are those for which different values are explored.

a semi-analytical hydrodynamical approach to study the combined effect of the stellar wind and orbital motion on the emitter, which is assumed to be one-dimensional (1D; see Molina \& Bosch-Ramon 2018; Molina et al. 2019, for a similar model in a microquasar scenario). The paper is structured as follows: the details of the model are given in Sect. 2, the results for a generic system are shown in Sect. 3, a specific application to the case of LS 5039 is done in Sect. 4, and a summary and a discussion are given in Sect. 5.

\section{Description of the general model}

As a representative situation, we study a binary system made of a massive O-type star and a pulsar that orbit around each other with a period of $T=5$ days. The orbit is taken circular for simplicity, with an orbital separation of $D=3 \times 10^{12} \mathrm{~cm} \approx 0.2 \mathrm{AU}$. The stellar properties are typical for a main sequence O-type star (Muijres et al. 2012), namely a temperature of $T_{\star}=40000 \mathrm{~K}$ and a luminosity of $L_{\star}=10^{39} \mathrm{erg} \mathrm{s}^{-1}$. For simplicity, we model the stellar wind as an isotropic supersonic outflow with a velocity of $v_{\mathrm{w}}=3 \times 10^{8} \mathrm{~cm} \mathrm{~s}^{-1}$ and a mass-loss rate of $\dot{M}_{\mathrm{w}}=$ $3 \times 10^{-7} M_{\odot} \mathrm{yr}^{-1}$. The wind velocity is taken constant and not following the classical $\beta$-law for massive stars (e.g., Pauldrach et al. 1986), as the effect of considering such a velocity profile for standard $\beta$ values is small for the purpose of this work. The pulsar has a spin-down luminosity of $L_{\mathrm{p}}=3 \times 10^{36} \mathrm{erg} \mathrm{s}^{-1}$, which is taken here as equal to the kinetic luminosity of the pulsar wind. The latter is assumed to be ultra-relativistic and isotropic, with Lorentz factor $\Gamma_{\mathrm{p}}=10^{5}$. The distance to the system is taken to be $d=3 \mathrm{kpc}$, and the inclination $i$ is left as a free parameter. A list of the parameter values used for a generic gamma-ray binary can be found in Table 1, and a sketch of the studied scenario is presented in Fig. 1. Throughout this section, the notation $u=\|\vec{u}\|$ is used to refer to a vector norm. Also, we use primed quantities in the fluid frame (FF), and unprimed ones in the frame of the star, assumed here to be the laboratory frame (LF).

\subsection{Dynamics}

The interaction of the stellar and the pulsar winds produces an interface region between the two where the shocked flow

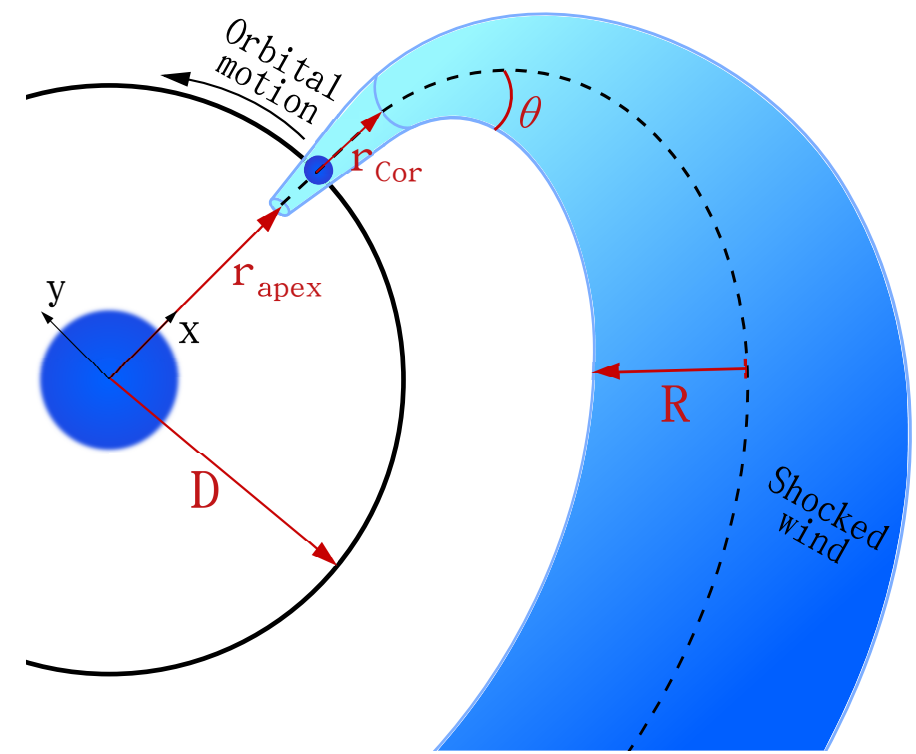

Fig. 1. Schematic zenithal view of the studied scenario (not to scale). Only a fraction of the orbit is shown for clarity.

pressures are in equilibrium: the so-called contact discontinuity (CD). Close to the binary system, where the orbital motion can be neglected, the shape of this surface is characterized by the pulsar-to-stellar wind momentum rate ratio, defined as

$\eta=\frac{L_{\mathrm{p}}}{\dot{M}_{\mathrm{w}} v_{\mathrm{w}} c}$

for $\Gamma_{\mathrm{p}} \gg 1$. The asymptotic half-opening angle of the CD can be approximated by the following expression from Eichler \& Usov (1993), which is in good agreement with numerical simulations (e.g., Bogovalov et al. 2008):

$\theta=28.6^{\circ}\left(4-\eta^{2 / 5}\right) \eta^{1 / 3}$

The apex of the CD, where the two winds collide frontally, is located along the star-pulsar direction at a distance from the star of $r_{\text {apex }}=D /(1+\sqrt{\eta})$, with $D$ being the orbital separation. For the adopted parameters, we obtain $\eta=0.018, \theta=28.7^{\circ}$, and $r_{\text {apex }}=0.88 D=2.6 \times 10^{12} \mathrm{~cm}$, the latter being constant owing to the circularity of the orbit.

The star is located at the origin of the coordinate system, which co-rotates with the pulsar. The $x$-axis is defined as the starpulsar direction, and the $y$-axis is perpendicular to it and points in the direction of the orbital motion. We model the evolution of the shocked stellar and pulsar winds in the inner interaction region as a straight conical structure of half-opening angle $\theta$ and increasing radius $R$, the onset of which is at $x=r_{\text {apex }}$, where it has a radius of $R_{0}=D-r_{\text {apex }}$, roughly corresponding to the characteristic size of the $\mathrm{CD}$ at its apex. The shocked flows are assumed to move along the $x$ direction in this region (i.e., the orbital velocity is neglected here with respect to the wind speed), until they reach a point where their dynamics start to be dominated by orbital effects, the Coriolis turnover. Beyond this point, the $\mathrm{CD}$ is progressively bent in the $-y$ direction due to the asymmetric interaction with the stellar wind, which arises from Coriolis forces (see Fig. 1 for an schematic view). As a result, the shocked flow structure acquires a spiral shape at large scales. One can estimate the distance of the Coriolis turnover to the pulsar $r_{\text {Cor }}$ by following the analytical prescription in Bosch-Ramon \& Barkov (2011), 
which comes from equating the total pulsar wind pressure to the stellar wind ram pressure due to the Coriolis effect (for $\Gamma_{\mathrm{p}} \gg 1$ ):

$\frac{L_{\mathrm{p}}}{4 \pi c r_{\mathrm{Cor}}^{2}}=\frac{\rho_{\mathrm{w}}(D)}{\left(1+r_{\mathrm{Cor}} / D\right)^{2}}\left(\frac{4 \pi}{T}\right)^{2} r_{\mathrm{Cor}}^{2}$,

where $\rho_{\mathrm{w}}(r)=\dot{M}_{\mathrm{w}} / 4 \pi r^{2} v_{\mathrm{w}}$ is the stellar wind density at a distance $\mathrm{r}$ from the star. Although approximate, this expression agrees well with results from 3D numerical simulations (BoschRamon et al. 2015). Numerically solving Eq. (3) for our set of parameters yields $r_{\text {Cor }}=0.94 D$. We note that in the case of an elliptical system $r_{\text {Cor }}$ changes along the orbit.

The radiation model explained in Sect. 2.2 focuses on the shocked pulsar material flowing inside the $\mathrm{CD}$. The values of the Lorentz factor of this fluid are set to increase linearly with distance from the CD apex $\left(\Gamma_{0}=1.06, v_{0}=c / 3\right)$ to the Coriolis turnover $(\Gamma=4, v=0.97 c)$, based on the simulations by Bogovalov et al. (2008). Beyond the Coriolis turnover, and due to some degree of mixing between the pulsar and stellar winds (see, e.g., the numerical simulations in Bosch-Ramon et al. 2015), we fix the shocked pulsar wind speed $v_{\text {Cor }}$ to a constant value that is left as a free parameter in our model, and could account for different levels of mixing. The shocked stellar wind that surrounds the shocked pulsar wind plays the role of a channel through which the former flows, and may have a much lower speed. This channel is assumed to effectively transfer the lateral momentum coming from the unshocked stellar wind to the shocked pulsar wind.

The trajectory of the shocked winds flowing away from the binary, which are assumed to form a (bent) conical structure, is determined by accounting for orbital motion and momentum balance between the upstream shocked pulsar wind and the unshocked stellar wind, with a thin shocked stellar wind channel playing the role of a mediator. This conical structure is divided into 2000 cylindrical segments of length $\mathrm{d} l=0.05 D$, amounting to a total length of $100 D$. Initially, all the shocked material moves along the $x$ axis between $x=r_{\text {apex }}$ and $x=D+r_{\text {Cor }}$. At the latter point, its position and momentum are, in Cartesian coordinates of the co-rotating frame,

$\vec{r}=\left(D+r_{\text {Cor }}, 0\right)$

$\vec{P}=(\dot{P} \mathrm{~d} t, 0)$,

where $\dot{P}$ is the momentum rate of the shocked flow, which we estimate as:

$\dot{P}=\frac{L_{\mathrm{p}}}{c}$.

For simplicity, we are not considering the contribution of the momentum of the stellar wind loaded through mixing into the pulsar wind before the Coriolis turnover. This contribution depends on the level of mixing, and could be as high as $\Omega \dot{M}_{\mathrm{w}} v_{\mathrm{w}}$, with $\Omega=R^{2} / 4\left(D+r_{\text {Cor }}\right)^{2}$ being the solid angle fraction subtended by the shocked pulsar wind at the Coriolis turnover, as seen from the position of the star. We note, nonetheless, that the inclusion of the stellar wind contribution to $\dot{P}$ has a modest impact on the radiation predictions of the model and can be neglected at this stage.

The unshocked stellar wind velocity in the co-rotating frame has components in both the $x$ and the $y$ directions:

$\overrightarrow{\hat{v}_{\mathrm{w}}}=v_{\mathrm{w}} \frac{(x, y)}{r}+\omega r \frac{(y,-x)}{r}$,

where $\omega$ is the orbital angular velocity, and the hat symbol is used to distinguish $\hat{v}_{\mathrm{w}}$ from the purely radial component of the wind, $v_{\mathrm{w}}$. In a stationary configuration, the force that the unshocked stellar wind exerts onto each segment of the shocked wind structure is

$\overrightarrow{F_{\mathrm{w}}}=\rho_{\mathrm{w}} \hat{v}_{\mathrm{w}}^{2} S \sin \alpha \frac{\overrightarrow{\hat{v}_{\mathrm{w}}}}{\hat{v}_{\mathrm{w}}}$,

with $\alpha$ being the angle between $\overrightarrow{\hat{v}}_{\mathrm{w}}$ and the shocked pulsar wind velocity $\vec{v}$, and $S \sin \alpha=2 R \mathrm{~d} l \sin \alpha$ the segment lateral surface normal to the stellar wind direction. The component of $\overrightarrow{F_{\mathrm{w}}}$ parallel to the fluid direction is assumed to be mostly converted into thermal pressure, whereas the perpendicular component $\overrightarrow{F_{\mathrm{w}}^{\perp}}=\overrightarrow{F_{\mathrm{w}}} \sin \alpha$ modifies the segment momentum direction. Thus, the interaction with the stellar wind only reorients the fluid, but it does not change its speed beyond the Coriolis turnover ${ }^{1}$.

We find the conditions for the subsequent segments by applying the following recursive relations:

$\vec{P}_{\mathrm{i}+1}=\vec{P}_{\mathrm{i}}+\vec{F}_{\mathrm{w}}^{\perp} \mathrm{d} t_{\mathrm{i}}$

$\vec{v}_{\mathrm{i}+1}=v_{\mathrm{i}} \frac{\vec{P}_{\mathrm{i}}}{P_{\mathrm{i}}}$

$\vec{r}_{\mathrm{i}+1}=\vec{r}_{\mathrm{i}}+\overrightarrow{v_{\mathrm{i}}} \mathrm{d} t_{\mathrm{i}}$

where $v_{\mathrm{i}}$ is the shocked pulsar wind velocity in each segment, and $\mathrm{d} t_{\mathrm{i}}=\mathrm{d} l / v_{\mathrm{i}}$ is the segment advection time. This procedure yields a fluid trajectory semi-quantitatively similar to that obtained from numerical simulations within approximately the first spiral turn (e.g., Bosch-Ramon et al. 2015).

\subsection{Characterization of the emitter}

The nonthermal emission is assumed to take place in the shocked pulsar wind, which moves through the shocked stellar wind channel and follows the trajectory defined in the previous section. We consider a nonthermal particle population consisting only of electrons and positrons. These are radiatively more efficient than accelerated protons (e.g., Bosch-Ramon \& Khangulyan 2009), but the presence of the latter cannot be discarded. Particles are accelerated at two different regions where strong shocks develop: the pulsar wind termination shock, located here at the $\mathrm{CD}$ apex; and the shock that forms in the Coriolis turnover (as in Zabalza et al. 2013, hereafter the Coriolis shock). In our general model, we consider for simplicity that both regions have the same power injected into nonthermal particles (in the LF), taken as a fraction of the total pulsar wind luminosity: $L_{\mathrm{NT}}=\eta_{\mathrm{NT}} L_{\mathrm{p}}$, with $\eta_{\mathrm{NT}}=0.1$. They also have the same acceleration efficiency $\eta_{\text {acc }}=0.1$, which defines the energy gain rate $\dot{E}_{\mathrm{acc}}^{\prime}=\eta_{\mathrm{acc}} e c B^{\prime}$ of particles for a given magnetic field $B^{\prime}$. The latter is defined through its energy density being a fraction of the total energy density at the CD apex (indicated with the subscript 0 ):

$\frac{B_{0}^{\prime 2}}{8 \pi}=\eta_{B} \frac{L_{\mathrm{p}}}{\pi R_{0}^{2} v_{0} \Gamma_{0}^{2}}$.

The magnetic field is assumed toroidal, and therefore it evolves along the emitter as $B^{\prime} \propto R^{-1} \Gamma^{-1}$. For simplicity, we assume

\footnotetext{
1 There must be some acceleration of the shocked flow away from the binary as a pressure gradient is expected, but for simplicity this effect is neglected here.
} 
that all the flow particles at the Coriolis turnover are reprocessed by the shock there, leading to a whole new population of particles. This means that the particle population beyond the Coriolis shock only depends on the properties of the latter, and is independent of the particle energy distribution coming from the initial shock at the CD apex. This assumption divides the emitter into two independent and distinct regions: one region between the $\mathrm{CD}$ apex and the Coriolis turnover (hereafter the inner region), and one beyond the latter (hereafter the outer region). We recall that the inner region has a velocity profile corresponding to a linear increase in $\Gamma$, whereas in the outer region the fluid moves at a constant speed (see Sect. 2.1).

To compute the particle evolution, the emitter is divided into 1000 segments of length $0.1 D$, in order to account for the same total length as in Sect 2.1. An electron and positron population is injected at each accelerator following a power-law distribution in the energy $E^{\prime}$, with an exponential cutoff and spectral index $p$ :

$Q^{\prime}\left(E^{\prime}\right) \propto E^{\prime p} \exp \left(-\frac{E^{\prime}}{E_{\max }^{\prime}}\right)$,

where $E_{\max }^{\prime}$ is the cutoff energy obtained by comparing the acceleration timescale, $t^{\prime}$ acc $=E^{\prime} /\left|\dot{E}_{\text {acc }}^{\prime}\right|$, with the cooling and diffusion timescales, $t_{\text {cool }}^{\prime}=E^{\prime} /\left|\dot{E}^{\prime}\right|$ and $t^{\prime}{ }_{\text {diff }}=3 R^{\prime 2} e B^{\prime} / 2 c E^{\prime}$, respectively ( $R$ is the perpendicular size and thus is taken $R^{\prime}=R$ ). We adopt $p=-2$ because it allows for a substantial power to be available for gamma-ray emission. Harder, and also a bit softer electron distributions would also be reasonable options. The particle injection is normalized by the total available power $L_{\mathrm{NT}}^{\prime}=$ $L_{\mathrm{NT}} / \Gamma^{2}$. We note that $E_{\max }^{\prime}$ and $L_{\mathrm{NT}}^{\prime}$ are not the same for both accelerators, since their properties differ. Particles are advected between subsequent segments following the bulk motion of the fluid (see Appendix B2 in de la Cita et al. 2016), and they cool down via adiabatic, synchrotron, and inverse Compton (IC) losses as they move along the emitter. The particle energy distribution at each segment is computed in the FF following the same recursive method as in Molina \& Bosch-Ramon (2018), which yields, for a given segment $\mathrm{k}$ :

$N_{\mathrm{k}}^{\prime}\left(E_{\mathrm{k}}^{\prime}\right)=N_{0}^{\prime}\left(E_{0}^{\prime}\right) \prod_{\mathrm{i}=\mathrm{k}}^{1} \frac{\dot{E}_{\mathrm{i}}^{\prime}\left(E_{\mathrm{i}-1}^{\prime}\right)}{\dot{E}_{\mathrm{i}}^{\prime}\left(E_{\mathrm{i}}^{\prime}\right)}$,

where $E_{\mathrm{k}}^{\prime}$ is the energy of a given particle at the location of segment $k$, and $E_{\mathrm{i}}^{\prime}$ is the energy that this same particle had when it was at the position of segment $i$, with $i \leq k$ (we note that $E_{\mathrm{i}}^{\prime}>E_{\mathrm{k}}^{\prime}$ due to energy losses).

For every point where we have the particle distribution, the synchrotron spectral energy distribution (SED) is computed following Pacholczyk (1970). The IC SED is obtained from the numerical prescription developed by Khangulyan et al. (2014) for a monodirectional field of stellar target photons with a blackbody spectrum. The distribution of electrons is isotropic in the FF. These SEDs are then corrected by Doppler boosting and absorption processes, the latter consisting of gamma-gamma absorption with the stellar photons (e.g. Gould \& Schréder 1967), and free-free absorption with the stellar wind ions (e.g., Rybicki \& Lightman 1986). We do not consider emission from secondary particles generated via the interaction of gamma rays with stellar photons, although it may have a nonnegligible impact on our results (see discussion in Sect. 5.2). Partial occultation of the emitter by the star is also taken into account, although it is only noticeable for very specific system-observer configurations. For a more detailed description of the SED computation we refer the reader to Molina et al. (2019).
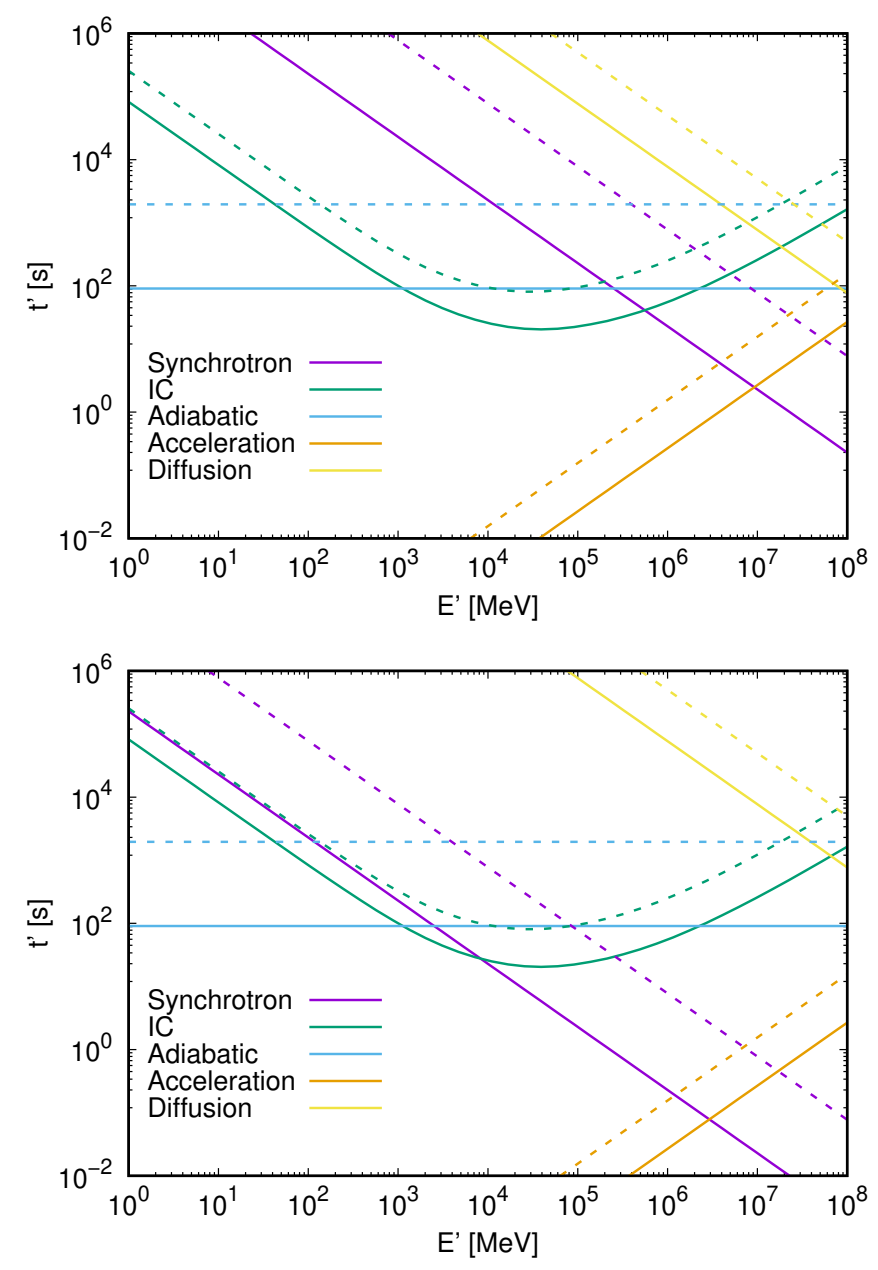

Fig. 2. Characteristic timescales in the FF for $v_{\mathrm{Cor}}=3 \times 10^{9}$, and $\eta_{B}=10^{-3}$ (top panel) and $10^{-1}$ (bottom panel). Solid and dashed lines represent the values at the $\mathrm{CD}$ apex and the Coriolis turnover locations, respectively.

\section{General results}

For the results presented in this section, we make use of the parameter values listed in Table 1 . The orbital phase $\Phi$ is defined such that the pulsar is in the inferior conjunction (INFC) for $\Phi=0$, and in the superior conjunction (SUPC) for $\Phi=0.5$.

\subsection{Energy losses and particle distribution}

Figure 2 shows the characteristic timescales in the FF for the cooling, acceleration, and diffusion processes, for $v_{\text {Cor }}=3 \times$ $10^{9} \mathrm{~cm} \mathrm{~s}^{-1}$, and $\eta_{B}=10^{-3}$ and $10^{-1}$, which correspond to initial magnetic fields of $B_{0}^{\prime}=4.15 \mathrm{G}$ and $41.5 \mathrm{G}$, respectively. In general, particle cooling is dominated by adiabatic losses at the lowest energies, IC losses at intermediate energies, and synchrotron losses at the highest ones unless a very small magnetic field with $\eta_{B}<10^{-5}$ is assumed. The exact energy values at which the different cooling processes dominate depend on $\eta_{B}$, and also on whichever emitter region we are looking at. Given the dependency of the synchrotron and acceleration timescales on the magnetic field, and that the latter decreases linearly with distance, $E_{\max }^{\prime}$ is higher at the Coriolis turnover than at the CD apex (see where the synchrotron and acceleration lines intersect in Fig. 2), allowing particles to reach higher energies at the location of the former. The larger region size involved and longer accumulation time, combined with the lower energy losses farther 


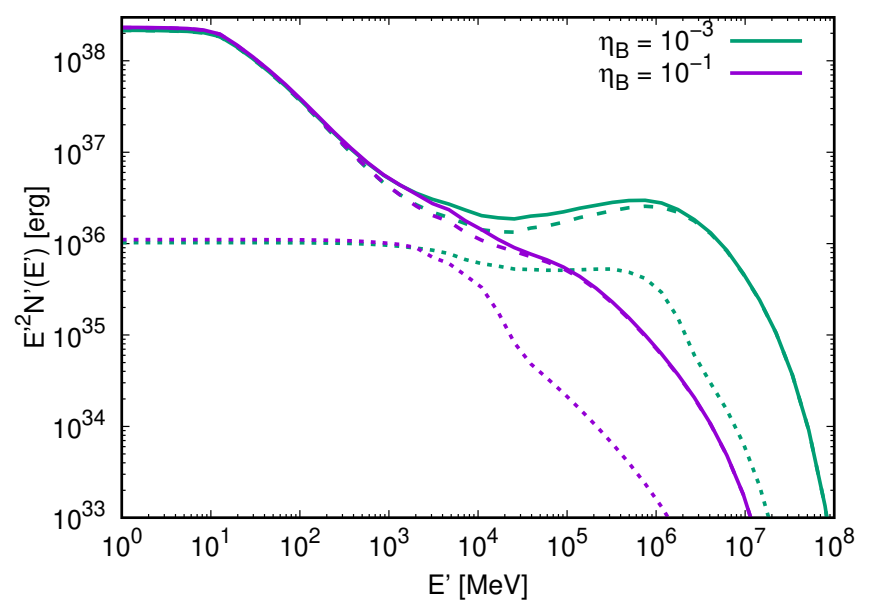

Fig. 3. Particle energy distribution in the FF for $v_{\text {Cor }}=3 \times 10^{9} \mathrm{~cm} \mathrm{~s}^{-1}$, and $\eta_{B}=10^{-3}$ (green lines) and $10^{-1}$ (purple lines). The contributions of the inner and outer regions are represented with dotted and dashed lines, respectively, and their sum is shown by the solid lines.

from the star, make the nonthermal particle distribution to be dominated by the outer region of the emitter, with just a small contribution from the inner part at middle energies, as seen in Fig. 3 (we recall that both regions are assumed to have the same injection power in the LF). The only significant effect of increasing the post-Coriolis shock speed to $v_{\text {Cor }}=10^{10} \mathrm{~cm} \mathrm{~s}^{-1}$ is the increase of the adiabatic losses by a factor of $\sim 3$ at the Coriolis turnover location and beyond (not shown in the figures). This results in a decrease of $N^{\prime}\left(E^{\prime}\right)$ for $E^{\prime} \lesssim 100 \mathrm{MeV}$, where adiabatic cooling (and particle escape) dominates.

\subsection{Spectral energy distribution}

The synchrotron and IC SEDs, as seen by the observer, are shown in Figs. 4 and 5 for $v_{\text {Cor }}=3 \times 10^{9} \mathrm{~cm} \mathrm{~s}^{-1}$ and $v_{\text {Cor }}=$ $10^{10} \mathrm{~cm} \mathrm{~s}^{-1}$, respectively. We take a representative orbital phase of $\Phi=0.3, i=60^{\circ}$, and $\eta_{B}=10^{-3}$ and $10^{-1}$. The overall SED has the typical shape for synchrotron and IC emission, with the magnetic field changing the relative intensity of each component. The IC SED is totally dominated by the outer region even if it is farther from the star and the target photon field is less dense than in the inner region. This happens because the former contains many more (accumulated) nonthermal particles that scatter stellar photons (see Fig. 3), and also because the ratio of synchrotron to IC cooling is smaller than in the inner region (therefore, more energy is emitted in the form of IC photons at the electron energies where radiative losses dominate). Synchrotron radiation, on the other hand, is more equally distributed between the two regions. We note, however, that Doppler boosting could make the inner region dominate both the IC and synchrotron emission in a broad energy range for orbital phases close to the INFC (see Sect. 3.3, and the discussion in Sect. 5.1).

\subsection{Orbital variability}

Light curves for two different system inclinations, $i=30^{\circ}$ and $60^{\circ}$, and post-Coriolis shock speeds, $v_{\text {Cor }}=3 \times 10^{9} \mathrm{~cm} \mathrm{~s}^{-1}$ and $10^{10} \mathrm{~cm} \mathrm{~s}^{-1}$, are shown in Figs. 6 and 7 for $\eta_{B}=10^{-3}$. Aside from a change in the flux normalization, the behavior of the light curves is very similar for $\eta_{B}=10^{-1}$. The modulation of X-rays is correlated with that of VHE gamma rays (top and bottom panels, respectively). Low-energy (LE) gamma rays (second panel) show a correlated modulation with VHE gamma rays and X-rays
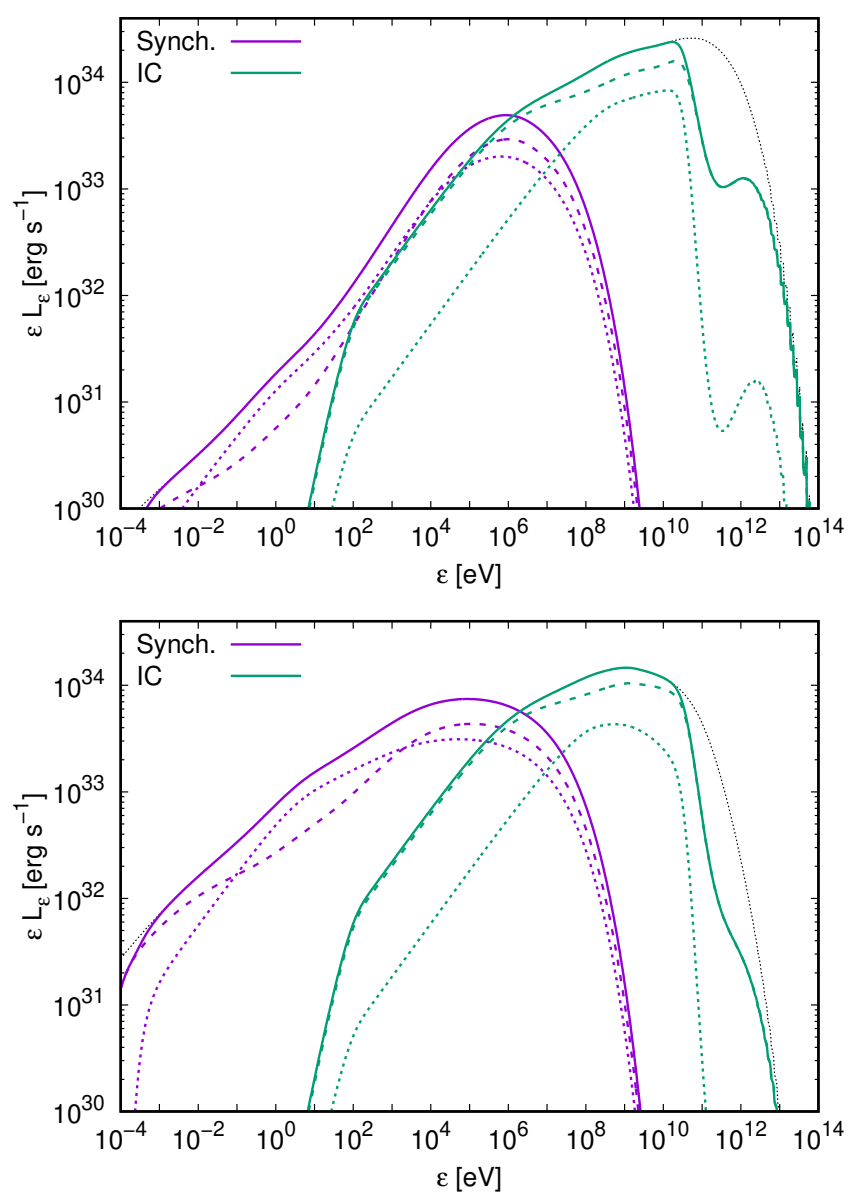

Fig. 4. Observer synchrotron (purple lines) and IC (green lines) spectral energy distributions for $\Phi=0.3, v_{\mathrm{Cor}}=3 \times 10^{9} \mathrm{~cm} \mathrm{~s}^{-1}, i=60^{\circ}$, and $\eta_{B}=10^{-3}$ (top panel) and $10^{-1}$ (bottom panel). The contributions of the inner and outer regions are represented with dotted and dashed lines, respectively. The black dotted lines show the total unabsorbed emission.

for $v_{\mathrm{Cor}}=10^{10} \mathrm{~cm} \mathrm{~s}^{-1}$, and an anti-correlated one for $v_{\mathrm{Cor}}=$ $3 \times 10^{9} \mathrm{~cm} \mathrm{~s}^{-1}$. This change in the LE gamma-ray modulation is caused by a higher boosting (deboosting) of the outer region emission close to the INFC (SUPC) for $v_{\text {Cor }}=10^{10} \mathrm{~cm} \mathrm{~s}^{-1}$, which overcomes the intrinsic IC modulation. This same effect is responsible for high-energy (HE) gamma rays (third panel) to not show a clear correlation with other energy bands at high $v_{\text {Cor }}$, whereas they are anti-correlated with VHE gamma rays and $\mathrm{X}$-rays (and correlated with LE gamma rays) at low $v_{\mathrm{Cor}}$. The fact that Doppler boosting modulates the emission in the opposite way as IC does also cause the predicted variability in the inner region as seen by the observer to significantly decrease with respect to its intrinsic one, whereas the effect on the outer region is less extreme due to a lower fluid speed. Asymmetries can be observed in the light curves due to the spiral trajectory of the emitter, although they are mild because most of the radiation is emitted within a distance of a few orbital separations from the star, where the spiral pattern is just beginning to form. The asymmetry only becomes more noticeable for HE gamma rays, $i=60^{\circ}$, and $v_{\text {Cor }}=10^{10} \mathrm{~cm} \mathrm{~s}^{-1}$ (third panel in Fig. 7), in which a double peak structure can be seen. The proximity to the star also makes VHE emission close to the SUPC to be almost suppressed by gamma-gamma absorption.

Figures 8 and 9 show, for $i=30^{\circ}$ and $60^{\circ}$ respectively, simulated radio sky maps at $5 \mathrm{GHz}$ for $v_{\mathrm{Cor}}=3 \times 10^{9} \mathrm{~cm} \mathrm{~s}^{-1}$, and $\eta_{B}=10^{-3}$ and $10^{-1}$. They are obtained by convolving the 

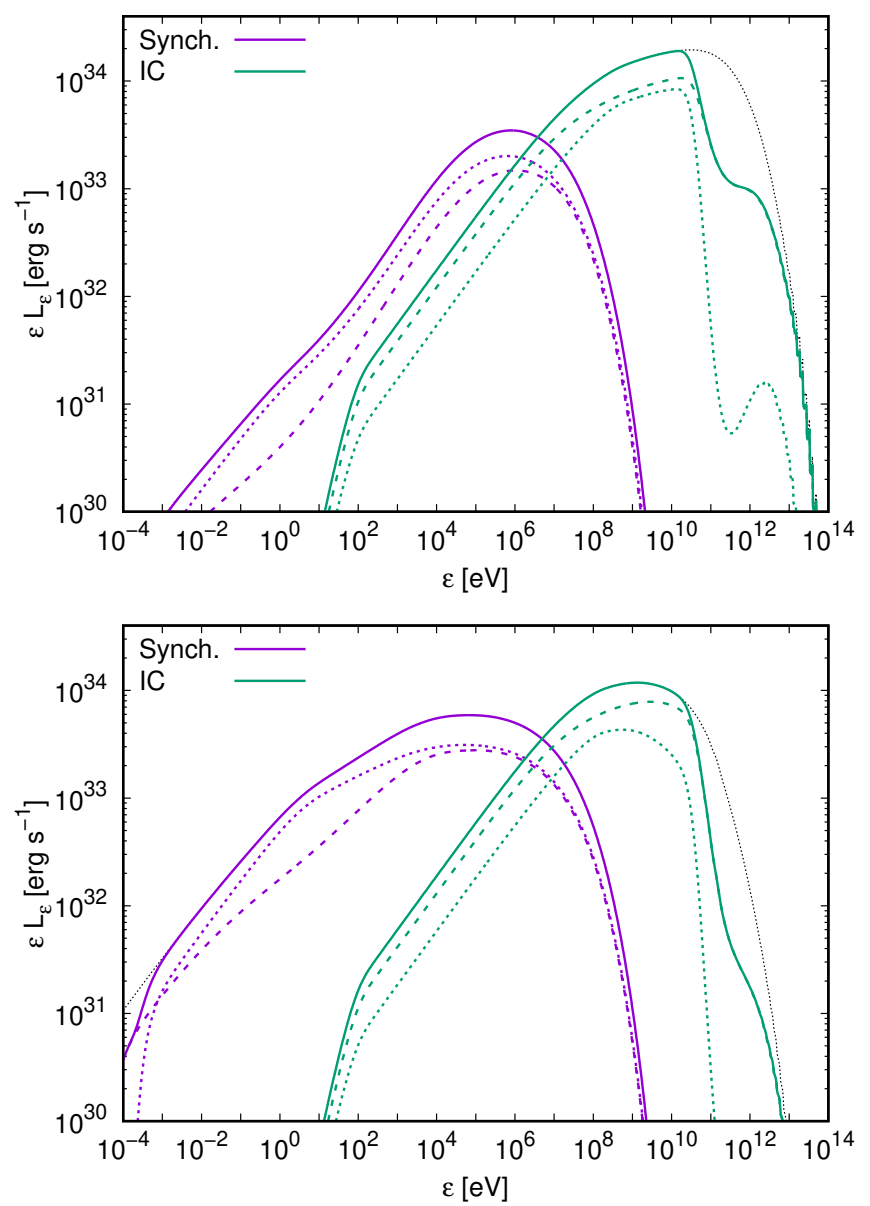

Fig. 5. Same as in Fig. 4, but for $v_{\text {Cor }}=10^{10} \mathrm{~cm} \mathrm{~s}^{-1}$.

projected emission of each segment with a 2D Gaussian with increasing width to approximately simulate the segment perpendicular extension. The resulting maps are then convolved again with a Gaussian telescope beam of full width at half maximum $(\mathrm{FWHM})=0.5$ mas. The contours are chosen so that the outermost one is $10 \mu \mathrm{Jy} \mathrm{beam}^{-1}$, on the order of the sensitivity of verylong-baseline interferometry (VLBI). Due to free-free absorption, radio emission from the inner region is highly suppressed, and what can be seen in the sky maps comes mostly from the outer region. In particular, the part of the outer region that contributes most to the radio emission is located close to the Coriolis shock, and has an angular size of $\sim 0.5$ mas, which corresponds to a linear size of $\sim 1 \mathrm{AU}$ at the assumed distance of $3 \mathrm{kpc}$. For high $\eta_{B}$ and the assumed angular resolution, the initial part of the spiral structure can be traced in the radio images, especially for low inclinations. For small $\eta_{B}$, only the sites very close to the Coriolis shock contribute to the emission due to the low synchrotron efficiency farther away, and hints of a spiral outflow cannot be seen. Regardless of the magnetic field value, the position of the maximum of the radio emission shifts for as much as $\approx 1$ mas at different orbital phases.

\section{Application to LS 5039}

LS 5039 is a widely studied binary system hosting a main sequence O-type star and a compact companion, the nature of which is still unclear. Low system inclinations $\left(i \lesssim 40^{\circ}\right)$ favor a black hole scenario, whereas higher inclinations favor the compact object to be a neutron star. Inclinations above $\approx 60^{\circ}$ are

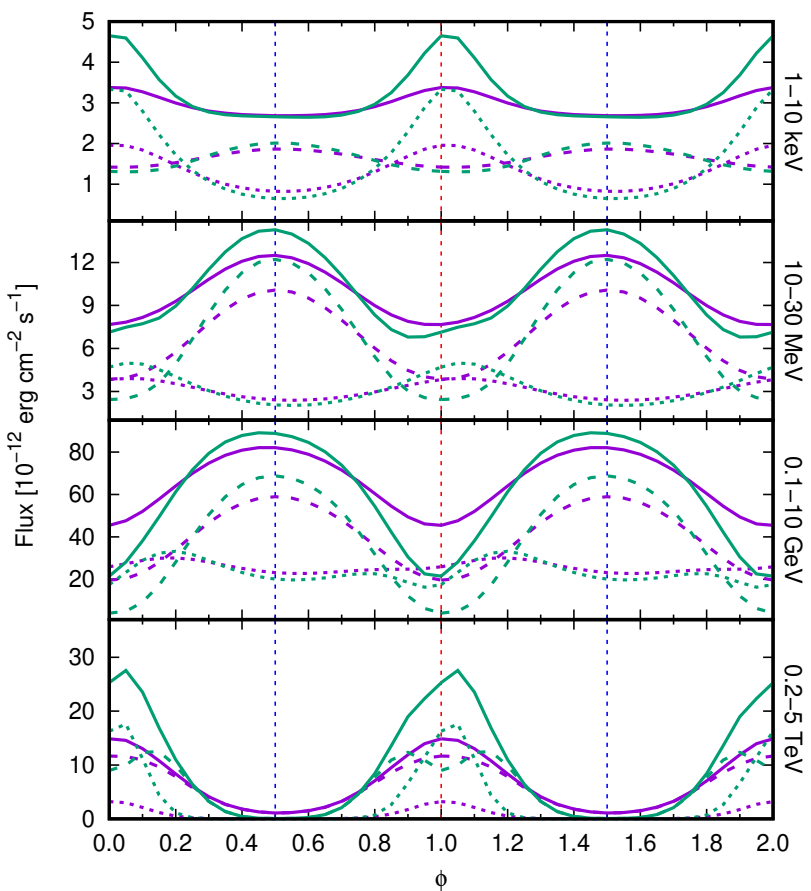

Fig. 6. Light curves at different energy ranges (indicated in the right side) for $\eta_{B}=10^{-3}, v_{\text {Cor }}=3 \times 10^{9} \mathrm{~cm} \mathrm{~s}^{-1}$, and $i=30^{\circ}$ (purple lines) and $60^{\circ}$ (green lines). The contributions from the inner and outer regions are shown with dotted and dashed lines, respectively. The vertical dotted blue and red lines show the position of the superior and inferior conjunctions, respectively. Two orbits are represented for a better visualization.

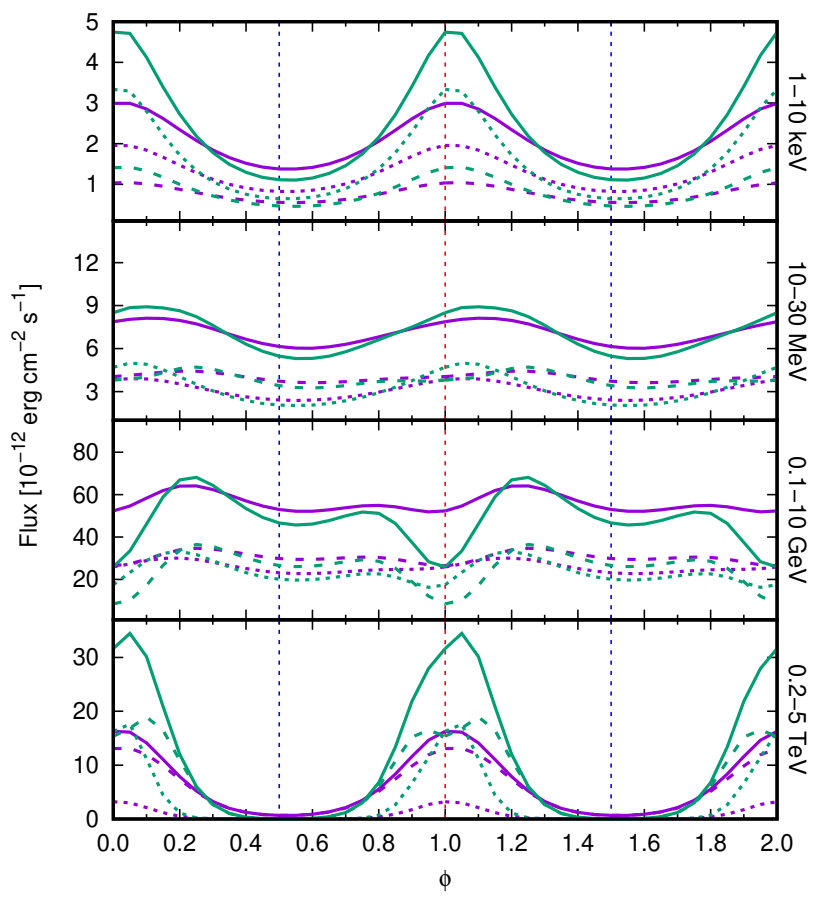

Fig. 7. Same as in Fig. 6, but for $v_{\text {Cor }}=10^{10} \mathrm{~cm} \mathrm{~s}^{-1}$.

unlikely due to the absence of X-ray eclipses in this system (Casares et al. 2005). LS 5039 has an elliptical orbit with a semimajor axis of $a \approx 2.4 \times 10^{12} \mathrm{~cm}$, an eccentricity of $e=0.35 \pm 0.04$, and a period of $T \approx 3.9$ days. The superior and inferior conjunctions are located at $\Phi=0.058$ and 0.716, respectively, with $\Phi=0$ corresponding to the periastron. The star has a 

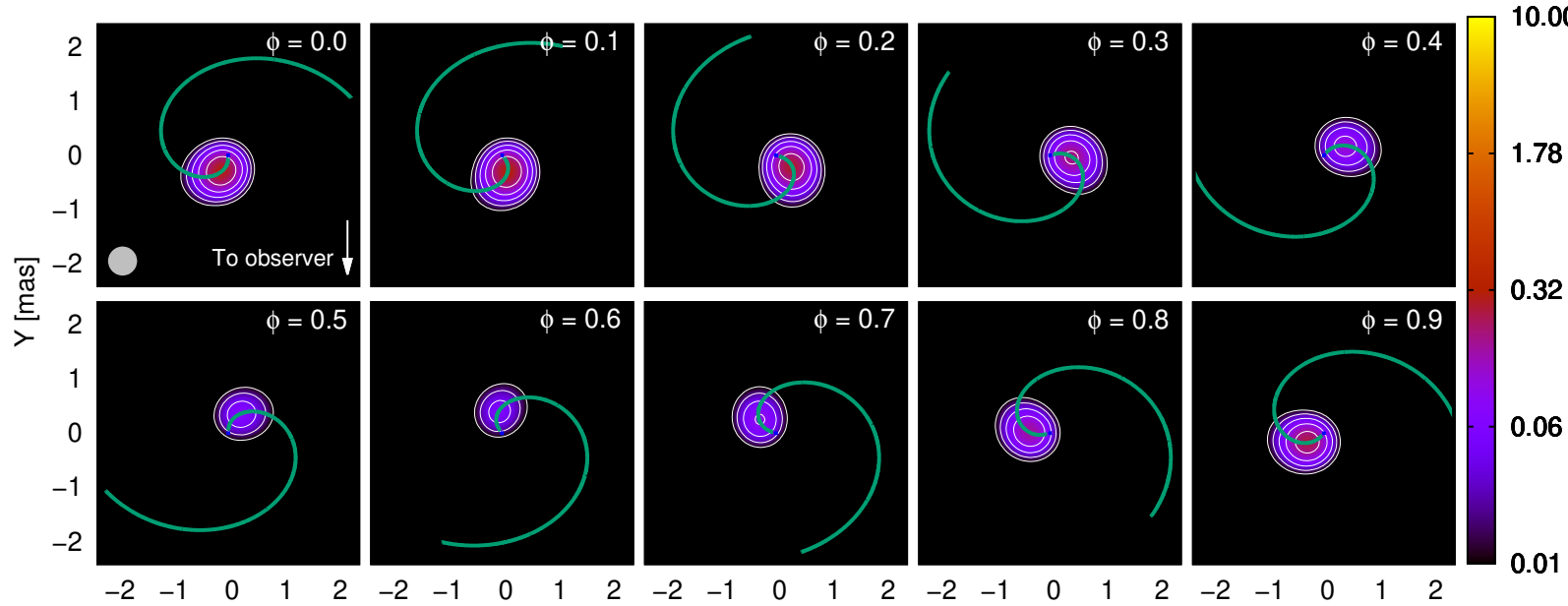

$\mathrm{X}$ [mas]
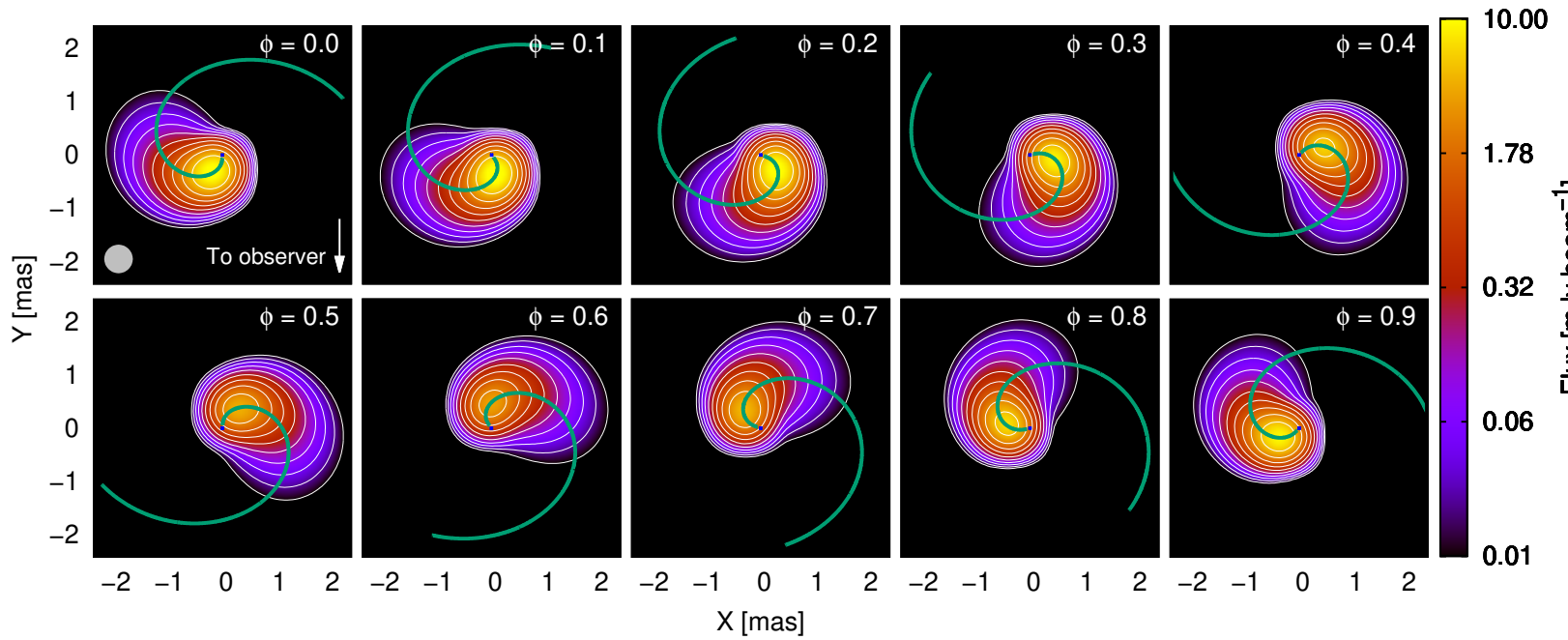

Fig. 8. Simulated radio sky maps at $5 \mathrm{GHz}$ for different orbital phases, $v_{\mathrm{Cor}}=3 \times 10^{9} \mathrm{~cm} \mathrm{~s}^{-1}, i=30^{\circ}$, and $\eta_{B}=10^{-3}$ (top panel) and $10^{-1}$ (bottom panel). The assumed telescope beam is shown as a gray circle in the bottom left corner of the first plot. The contour lines start at a flux of $10 \mu \mathrm{Jy}$ beam $^{-1}$ and increase with a factor of 2 . The star is represented (to scale) with a blue circle at $(0,0)$, and the solid green line shows the axis of the conical emitter, the onset of which points toward the observer for $\Phi=0$, and opposite to it for $\Phi=0.5$.

luminosity of $L_{\star}=(7 \pm 1) \times 10^{38} \mathrm{erg} \mathrm{s}^{-1}$, a radius of $R_{\star}=9.3 \pm$ $0.7 R_{\odot}$, and an effective temperature of $T_{\star}=(3.9 \pm 0.2) \times 10^{4} \mathrm{~K}$ (Casares et al. 2005). The stellar mass-loss rate obtained through $\mathrm{H} \alpha$ measurements is in the range $\dot{M}_{\mathrm{w}}=3.7-4.8 \times 10^{-7} M_{\odot} \mathrm{yr}^{-1}$ (Sarty et al. 2011), although this value would be overestimated if the wind were clumpy (e.g., Muijres et al. 2011). The assumption of an extended X-ray emitter (as it is the case in this work) places an upper limit for $\dot{M}_{\mathrm{w}}$ of up to a few times $10^{-7} M_{\odot} \mathrm{yr}^{-1}$, with the exact value depending on the system parameters (Szostek \& Dubus 2011; see also Bosch-Ramon et al. 2007). The lack of thermal X-rays in the shocked stellar wind, in the context of a semi-analytical model of the shocked wind structure, puts an upper limit in the putative pulsar spin down luminosity of $L_{\mathrm{p}} \leq 6 \times 10^{36} \mathrm{erg} \mathrm{s}^{-1}$ (Zabalza et al. 2011). The latest Gaia DR2 parallax data (Gaia Collaboration 2018; Luri et al. 2018) sets a distance to the source of $d=2.1 \pm 0.2 \mathrm{kpc}$. The rest of the model parameters are unknown for LS 5039.

Carrying out a statistical analysis is hardly possible in our context, as we have many free parameters or parameters that are loosely constrained. Thus, we have looked for a set of parameter values that approximately reproduce the observational data, but the result should be considered just as illustrative of the model capability to reproduce the source behavior, and not a fit. We note that, for this purpose, we use a different value of the nonthermal power fraction for each accelerator $\left(\eta_{\mathrm{NT}}^{A}\right.$ for the $\mathrm{CD}$ apex, and $\eta_{\mathrm{NT}}^{B}$ for the Coriolis shock). Table 2 shows all the model parameters used for the study of LS 5039, which are left constant throughout the whole orbit. For these parameters, we obtain $\eta=0.035, \theta=35.5^{\circ}, 0.55 a \leq r_{\text {apex }} \leq 1.14 a$, and $1.03 a \leq r_{\text {Cor }} \leq 1.29 a$, with the lower (upper) limits corresponding to the periastron (apastron).

Figure 10 shows, for $i=60^{\circ}$, the computed SED averaged over two wide phase intervals, one around the INFC $(0.45<$ $\Phi \leq 0.90)$, and the other one around the SUPC $(0.90<\Phi$ or $\Phi \leq 0.45$ ). Observational data points of Suzaku (Takahashi et al. 2009), COMPTEL (Collmar \& Zhang 2014), Fermi/LAT (Fermi LAT Collaboration 2009; Hadasch et al. 2012), and H.E.S.S. (Aharonian et al. 2006) averaged over the same phase intervals are also plotted. The SEDs for different system inclinations in which a pulsar scenario is viable $\left(40^{\circ} \lesssim i \lesssim 60^{\circ}\right)$ are not represented due their similarity to the one shown in Fig. 10. Synchrotron emission dominates for $\varepsilon \lesssim 10 \mathrm{GeV}$, with IC only contributing significantly at VHE. With the exception of the COMPTEL energies $(1 \mathrm{MeV} \lesssim \varepsilon \lesssim 30 \mathrm{MeV})$, the SED reproduces reasonably well the magnitude of the observed fluxes, especially for X-rays and VHE gamma rays. At energies 

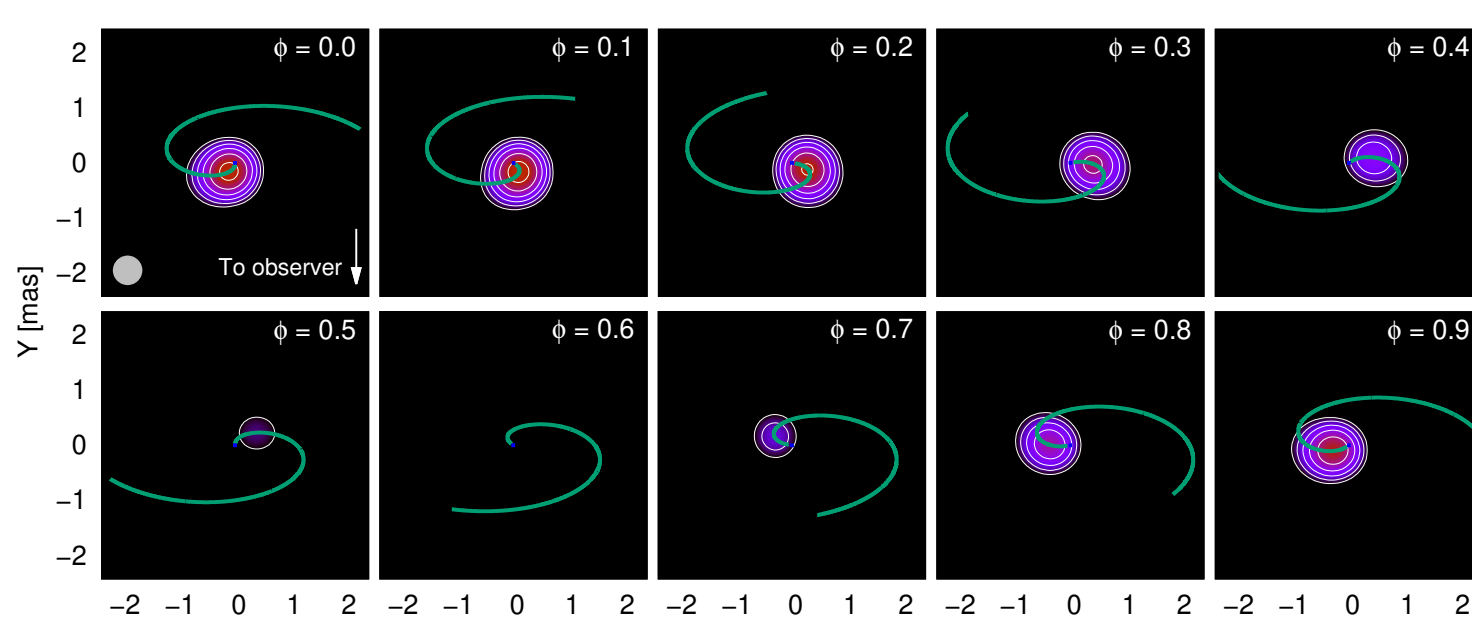

10.00
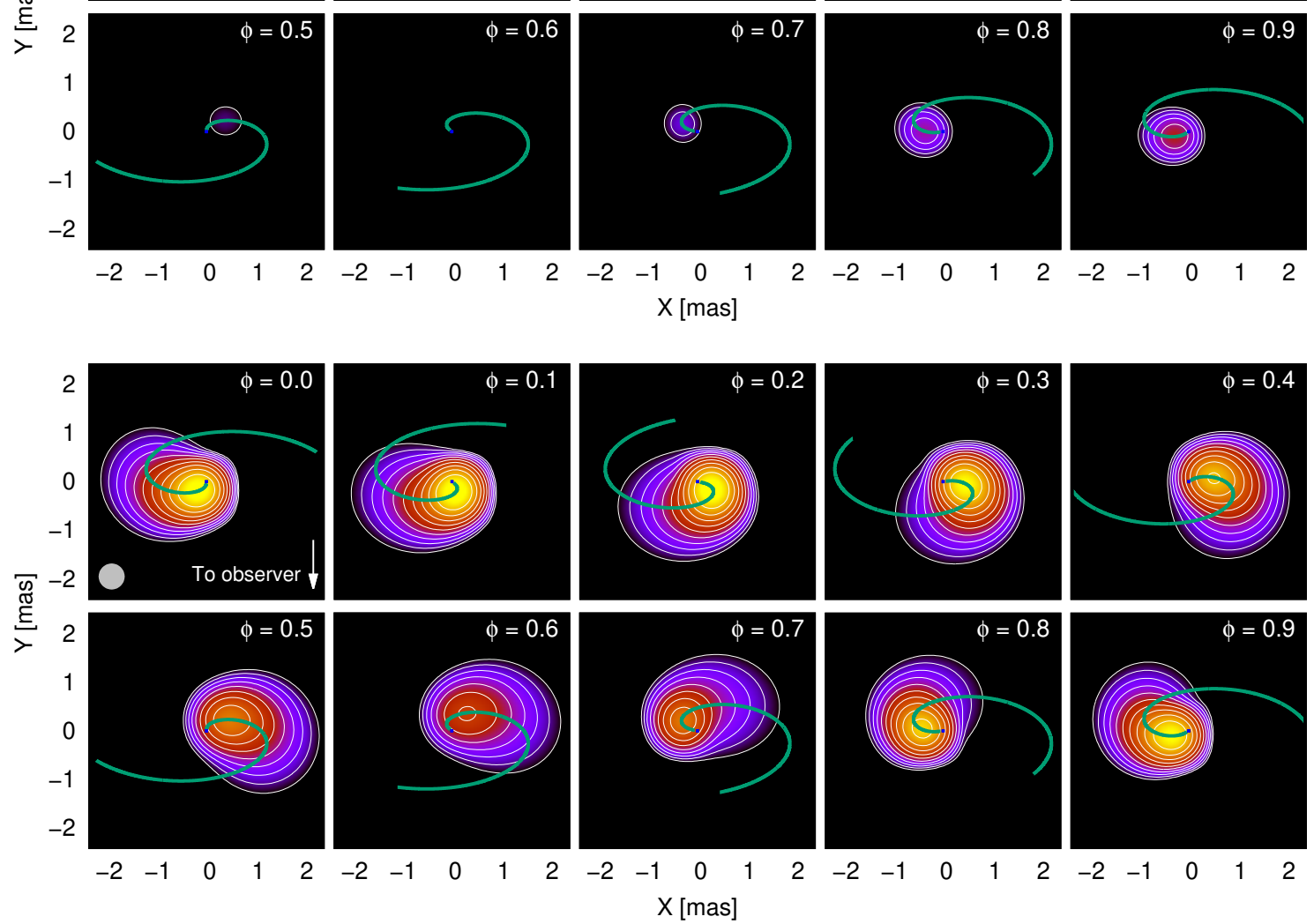

10.00
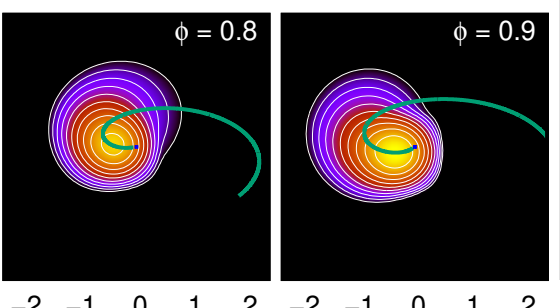

Fig. 9. Same as in Fig. 8, but for $i=60^{\circ}$.

of $100 \mathrm{MeV} \lesssim \varepsilon \lesssim 10 \mathrm{GeV}$, the model overpredicts (underpredicts) the emission around INFC (SUPC). The hard electron spectrum allows the IC component not to strongly overestimate the fluxes around $10 \mathrm{GeV}$, although we must note that IC emission from secondary pairs, not taken into account, may increase a bit the predicted fluxes.

The computed LS 5039 light curves are shown in Fig. 11 for the limits of the system inclination range allowed for a pulsar binary system $\left(40^{\circ} \lesssim i \lesssim 60^{\circ}\right)$. Most of the emission comes from the outer region, regardless of the energy range. As in the SED, the model matches well the Suzaku and H.E.S.S observations, except for an underestimation of the latter fluxes around the SUPC. Inclinations close to $60^{\circ}$ are favored by the presence of a double peak in the VHE fluxes, which is not reproduced for lower values of $i$. This double peak is originated by the effect of the system orientation in the IC emission and Doppler boosting; the peak at $\Phi \approx 0.5$ has a higher intrinsic IC emission, but a lower boosting than the peak at $\Phi \approx 0.85$. At Fermi/LAT energies, the model predicts a maximum in the light curve around the INFC and a minimum around the SUPC, contrary to what is observed. This happens because, in this energy range, the model emission is dominated by synchrotron radiation, which has a maximum around the INFC due to Doppler boosting. In the COMPTEL energy range, the relative behavior of the computed light curve is similar to the observations, although a factor of 4-5 lower.
Table 2. Parameters used for the study of LS 5039.

\begin{tabular}{lcc}
\hline \hline & Parameter & Value \\
\hline \multirow{2}{*}{ Star } & Temperature $T_{\star}$ & $4 \times 10^{4} \mathrm{~K}$ \\
& Luminosity $L_{\star}$ & $7 \times 10^{38} \mathrm{erg} \mathrm{s}^{-1}$ \\
& Mass-loss rate $\dot{M}_{\mathrm{w}}$ & $1.5 \times 10^{-7} M_{\odot} \mathrm{yr}^{-1}$ \\
& Wind speed $v_{\mathrm{w}}$ & $3 \times 10^{8} \mathrm{~cm} \mathrm{~s}^{-1}$ \\
\hline Pulsar & Luminosity $L_{\mathrm{p}}$ & $3 \times 10^{36} \mathrm{erg} \mathrm{s}^{-1}$ \\
& Wind Lorentz factor $\Gamma_{\mathrm{p}}$ & $10^{5}$ \\
\hline \multirow{2}{*}{ System } & Orbit semi-major axis $a$ & $2.4 \times 10^{12} \mathrm{~cm}$ \\
& Orbital period $T$ & $3.9 \mathrm{days}$ \\
& Orbital eccentricity $e$ & 0.35 \\
& Distance to the observer $d$ & $2.1 \mathrm{kpc}$ \\
& CD apex NT fraction $\eta_{\mathrm{NT}}^{A}$ & 0.03 \\
& Cor. shock NT fraction $\eta_{\mathrm{NT}}^{B}$ & 0.18 \\
& Acceleration efficiency $\eta_{\mathrm{acc}}$ & 0.8 \\
Injection power-law index $p$ & -1.3 \\
& Coriolis turnover speed $v_{\mathrm{Cor}}$ & $3 \times 10^{9} \mathrm{~cm} \mathrm{~s}^{-1}$ \\
Magnetic fraction $\eta_{B}$ & 0.02 \\
& System inclination $i$ & $40^{\circ}, 60^{\circ}$ \\
\hline
\end{tabular}

Figure 12 shows the computed radio sky map of LS 5039 at $5 \mathrm{GHz}$, for $i=60^{\circ}$ and a telescope beam with $F W H M=0.5$ mas. 


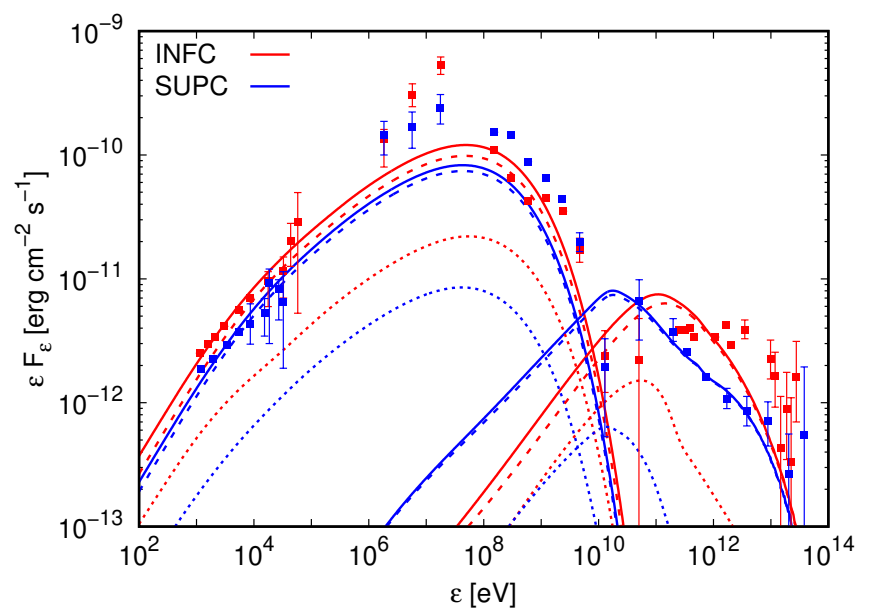

Fig. 10. Observer synchrotron and IC SEDs of LS 5039 for $i=60^{\circ}$, averaged over the INFC (red lines; $0.45<\Phi \leq 0.90$ ) and SUPC (blue lines; $0.90<\Phi$ or $\Phi \leq 0.45$ ) phase intervals. Dotted and dashed lines represent the contributions of the inner and outer regions, respectively. From left to right, data from Suzaku, COMPTEL, Fermi/LAT, and H.E.S.S. are also represented.

The sky map for $i=40^{\circ}$ is very similar and is not shown. Since we do not consider particle reacceleration beyond the Coriolis shock, these maps show the synchrotron emission up to a few orbital separations from the latter, as farther away synchrotron emission is too weak to significantly contribute to the radio flux. This lack of reacceleration does not allow for a meaningful comparison between our model and the observations. While the predicted total radio flux at $5 \mathrm{GHz}$, averaged over a whole orbit, is $0.10 \mathrm{mJy}$, the detected one is around $20 \mathrm{mJy}$ (Moldón et al. 2012). The assumption of a hard particle spectrum $(p=-1.3)$ needed to explain the SEDs makes most of the available power to go into the most energetic electrons and positrons. Therefore, only a small part of the energy budget goes to those lower energy particles responsible for the radio emission, which makes the latter considerably fainter than in the generic case studied in Sect. 3, in which $p=-2$. Despite the almost point-like and faint nature of the radio source, the emission maximum is displaced along the orbit by a similar angular distance of $\approx 1$ mas.

\section{Summary and discussion}

We have developed a semi-analytical model, consisting of a 1D emitter, which can be used to describe both the dynamics and the radiation of gamma-ray binaries in a colliding wind scenario that includes orbital motion. In the following, we discuss the obtained results for a generic system and for the specific case of LS 5039, as well as the main sources of uncertainty.

\subsection{General case}

In general, a favorable combination of nonradiative and radiative losses, and residence time of the emitting particles, leads to an outer emitting region that is more prominent in its nonthermal energy content and emission than the inner region. Thus, the SEDs are dominated by the outer region for most orbital phases. Moreover, both radio and VHE gamma-ray emission are suppressed close to the star due to free-free and gamma-gamma absorption, respectively, unless small system inclinations $i<30^{\circ}$ and/or orbital phases close to the INFC are considered. Nevertheless, the light curves show a nonnegligible or even dominant contribution of the inner region close to the INFC for a

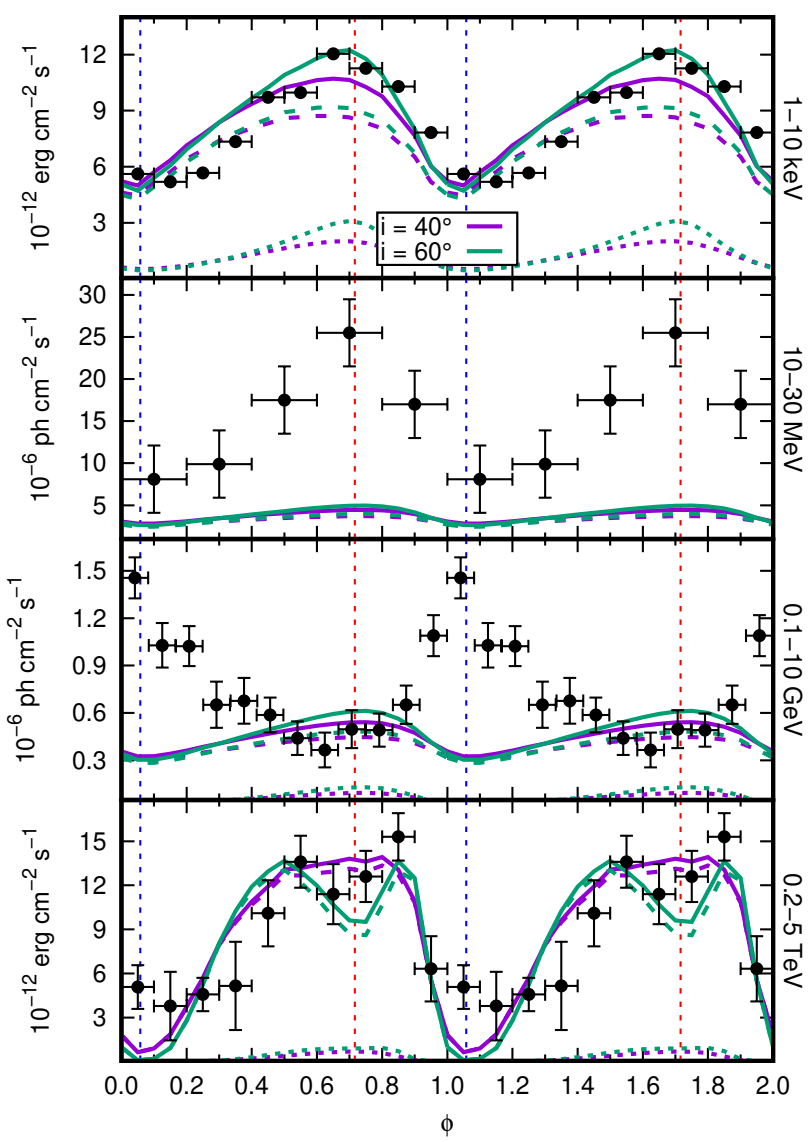

Fig. 11. From top to bottom: light curves of LS 5039 in the Suzaku (1-10 keV), COMPTEL $(10-30 \mathrm{MeV})$, Fermi/LAT $(0.1-10 \mathrm{GeV})$, and H.E.S.S. $\left(0.2-5 \mathrm{TeV}\right.$ ) energy ranges, for $i=40^{\circ}$ (purple lines) and $60^{\circ}$ (green lines). The contributions of the inner and outer regions are shown with dotted and dashed lines, respectively. The phases corresponding to the INFC (SUPC) are shown with red (blue) vertical dashed lines. Flux units are not the same for all the plots.

broad energy range. This is mainly caused by the inner region emission being more Doppler-boosted than the outer region one, due to the flow moving faster in the former. Higher values of $v_{\text {Cor }}$ (which could indicate a lower degree of mixing of stellar and pulsar winds) tend to decrease the radiative output of the outer region due to an increase in the adiabatic losses and in the escape rate of particles from the relevant emitting region. This trend is only broken for orbital phases close to the INFC, where a higher Doppler boosting is able to compensate for the decrease of intrinsic emission in the outer region. Doppler boosting (and hence the adopted velocity profile) has also a high influence on the orbital modulation of the IC radiation in both the inner and outer regions, to the point that emission peaks can become valleys owing to a change in the fluid speed of a factor of $\sim 3$ in the outer region $^{2}$.

Radio emission could be used to track part of the spiral trajectory of the shocked flow for strong enough magnetic fields with $\eta_{B} \gtrsim 0.1$, while no evidence of such spiral structure is predicted for low fields. In any case, as long as the overall radio emission is detectable, variations of the image centroid position on the order of 1 mas (for a distance to the source of $\sim 3 \mathrm{kpc}$ ) could be used as an indication of the dependency of the emitter structure with the orbital phase. This behavior is not exclusive

\footnotetext{
2 A similar effect is expected to happen if different velocity profiles are assumed for the inner region, but this has not been explicitly explored in this work.
} 

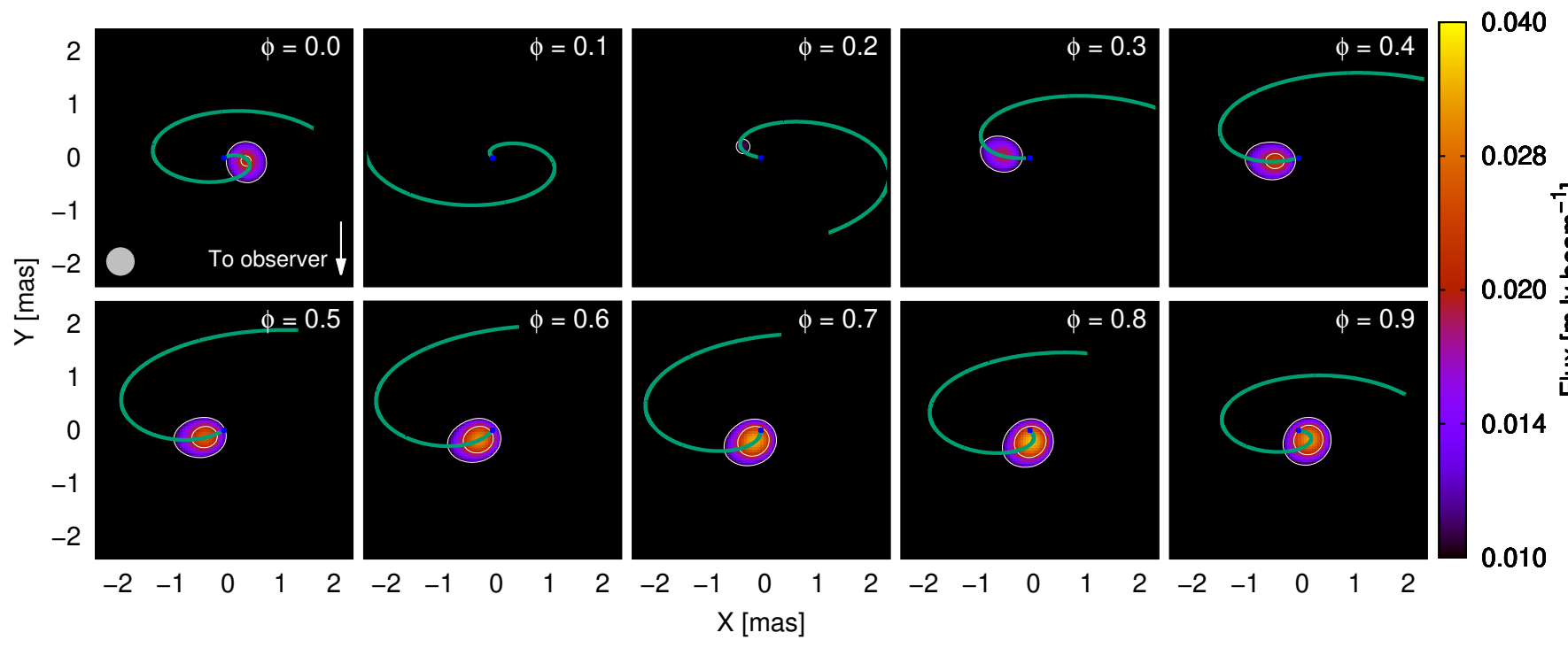

Fig. 12. Same as in Fig. 9, but for the LS 5039 model parameters, with $i=60^{\circ}$. Note the change in the color scale.

of a colliding wind scenario, however, since the jets in a microquasar scenario could be affected by orbital motion in a similar manner (e.g., Bosch-Ramon 2013; Molina \& Bosch-Ramon 2018; Molina et al. 2019).

There are some limitations in our model that should be acknowledged. One of them is the simplified dynamical treatment of the emitter, considered as a 1D structure. Not using proper hydrodynamical simulations makes the computed trajectory approximately valid within the first spiral turn, after which strong instabilities would significantly affect the fluid propagation, as seen in Bosch-Ramon et al. (2015). Nonetheless, since most of the emission comes from the regions close to the binary system, we do not expect strong variations in the radiative output due to this issue. We are not considering particle reacceleration beyond the Coriolis shock, although additional shocks and turbulence are expected to develop under the conditions present farther downstream (Bosch-Ramon et al. 2015), and these processes could contribute to increase the nonthermal particle energetics. Accounting for reacceleration would in turn increase the emission farther from the binary system, resulting for instance in more extended radio structures, although with the aforementioned inaccuracies in the trajectory computation gaining more importance.

Finally, we note that the model results for a generic case could change significantly for different values of some parameters that are difficult to determine accurately. The fluid speed and detailed geometry in the inner and outer regions are hard to constrain observationally. Precise values of the magnetic field, the electron injection index, the acceleration efficiency, and the nonthermal luminosity fraction are also difficult to obtain due to the presence of some degeneracy among them. Any significant changes in these quantities with respect to the values adopted in this work could have a strong influence on the emission outputs of the system.

\subsection{LS 5039}

Several model parameters can be fixed for the study of LS 5039 thanks to the existing observations of the source. Those parameters that cannot be obtained from observations are determined by heuristically (although quite thoroughly) exploring the parameter space, trying to better reproduce the observed LS 5039 emission. For this purpose, a hard particle spectrum is injected at both accelerators, with a power-law index $p=-1.3$, and a very high acceleration efficiency of $\eta_{\text {acc }}=0.8$ is assumed in both locations (we note that values between 0.5 and 1 give qualitatively similar results). These values are higher than those used for the general case, already quite extreme, although they cannot be discarded given the current lack of knowledge on the acceleration processes taking place in LS 5039 (or even the emitting process; see, e.g., Khangulyan et al. 2020, for an alternative origin of the gamma rays in which a synchrotron-related mechanism does play a role). Additionally, since the outer region behavior reproduces better the observed (X-ray and VHE) light curves, a higher $\eta_{\mathrm{NT}}$ is assumed for the Coriolis shock than for the CD apex, providing the former with a larger nonthermal luminosity budget (i.e., $\eta_{\mathrm{NT}}=0.18$ versus 0.03 ).

The model nicely reproduces the observed X-ray and VHE gamma-ray emission of LS 5039, as well as the HE gamma-ray flux, although it fails to properly account for the HE gammaray modulation due to the synchrotron dominance in this energy range. A possible solution to this issue could be the inclusion of particle reacceleration beyond the Coriolis shock, which combined with the lower magnetic field may result in enough IC emission to explain the observed modulation of the HE gamma rays. This would also alleviate the extreme value of $\eta_{\text {acc }}$ needed for the synchrotron emission to reach $\mathrm{GeV}$ energies. The largest difference between the model predictions and the observations comes at photon energies around $10 \mathrm{MeV}$, where the emission is underestimated by a factor of up to 5 (although interestingly the modulation is as observed). Some MeV flux would be added if we accounted for the synchrotron emission of electron-positron pairs created by gamma rays interacting with stellar photons (e.g., Bosch-Ramon et al. 2008; Cerutti et al. 2010), which is not included in our model. However, this component cannot be much larger than the $\mathrm{TeV}$ emission, as otherwise IC from these secondary particles would largely violate the $10 \mathrm{GeV}$ observational flux constraints. Therefore, the energy budget of secondaries alone cannot explain the MeV flux.

The lack of predicted VHE emission around the SUPC is due to strong gamma-gamma absorption. The fact that we use a 1D emitter at the symmetry axis of the conical CD overestimates 
this absorption, since we are not considering emitting sites at the CD itself, which is approximately at a distance $R$ from the axis (see Fig. 1) or even farther (see the shocked pulsar wind extension in the direction normal to the orbital plane in the corresponding maps in Bosch-Ramon et al. 2015). Within a few orbital separations from the pulsar (where most of the emission comes from), the CD is significantly farther from the star than its axis, and the observer, star, and flow relative positions are also quite different, allowing for lower absorption in some of the emitting regions. Therefore, the use of an extended emitter would reduce gamma-gamma absorption and increase the predicted VHE fluxes around the SUPC, possibly explaining the H.E.S.S. fluxes. Particle reacceleration beyond the Coriolis shock may also make some regions farther away from the star to emit VHE photons that would be less absorbed. As already discussed for the general case, reacceleration would also extend the radio emission and, if accounted for, could allow for a sky map comparison with VLBI observations (e.g., Moldón et al. 2012). Thus, one could constrain a reacceleration region added to our model using VLBI observations, but this is beyond the scope of the present work. It is worth noting that emission from secondary particles could also have a significant contribution to the extended and nonextended VLBI components (Bosch-Ramon \& Khangulyan 2011).

Our computed SED is qualitatively different to the best fit scenario presented in del Palacio et al. (2015), in which a onezone model was applied for an accelerator in a fixed position at a distance of $1.4 a \approx 3.3 \times 10^{12} \mathrm{~cm}$ from the star. Although the general shape is similar, their SED is totally dominated by IC down to $\sim 1 \mathrm{MeV}$, whereas in our model IC is only relevant at energies $\gtrsim 10 \mathrm{GeV}$. Another one-zone model in Takahashi et al. (2009) explains well the X-ray and VHE gamma-ray emission and modulation, although it underestimates the fluxes at $\mathrm{MeV}$ and $\mathrm{GeV}$ energies (which were not available by the time of publication of this work). Our synchrotron and IC phase-averaged SEDs above $100 \mathrm{MeV}$ are somewhat close to those in Zabalza et al. (2013). They applied a two-zone model to LS 5039, with the two emitting regions located at the CD apex and the Coriolis shock. Their model reproduces better the GeV modulation, although it also fails to explain the $\mathrm{MeV}$ emission, and underestimates the X-ray emission by more than one order of magnitude. Similar results were obtained by Dubus et al. (2015) with a model that computes the flow evolution through a 3D hydrodynamical simulation of the shocked wind close to the binary system, where orbital motion is still unimportant (and thus, it does not include the Coriolis shock). Modulation in the HE band is well explained there, although both the X-ray and the $\mathrm{MeV}$ emission are underestimated. All of the above, added to the fact that the 1D emitter model presented in this work also fails to reproduce some of the LS 5039 features, seems to point toward the need of more complex models to describe the behavior of this source, accounting for particle reacceleration, using data from 3D (magneto-)hydrodynamical simulations to compute the evolution of an emitter affected by orbital motion, and possibly including the unshocked pulsar wind zone to correctly describe the $\mathrm{MeV}$ radiation (see, e.g., Derishev et al. 2012).

Acknowledgements. We would like to thank the referee for his/her constructive and useful comments, which were helpful to improve the manuscript. We acknowledge support by the Spanish Ministerio de Economía y Competitividad (MINECO/FEDER, UE) under grant AYA2016-76012-C3-1-P, with partial support by the European Regional Development Fund (ERDF/FEDER), and from the Catalan DEC grant 2017 SGR 643. EM acknowledges support from MINECO through grant BES-2016-076342.

\section{References}

Abeysekara, A. U., Benbow, W., Bird, R., et al. 2018, ApJ, 867, L19 Aharonian, F., Akhperjanian, A. G., Aye, K. M., et al. 2005, A\&A, 442, 1 Aharonian, F., Akhperjanian, A. G., Bazer-Bachi, A. R., et al. 2006, A\&A, 460, 743

Aharonian, F. A., Akhperjanian, A. G., Bazer-Bachi, A. R., et al. 2007, A\&A, 469, L1

Barkov, M. V., \& Bosch-Ramon, V. 2016, MNRAS, 456, L64

Bogovalov, S. V., Khangulyan, D. V., Koldoba, A. V., Ustyugova, G. V., \& Aharonian, F. A. 2008, MNRAS, 387, 63

Bosch-Ramon, V. 2013, Eur. Phys. J. Web Conf., 61, 03001

Bosch-Ramon, V., \& Barkov, M. V. 2011, A\&A, 535, A20

Bosch-Ramon, V., \& Khangulyan, D. 2009, Int. J. Mod. Phys. D, 18, 347

Bosch-Ramon, V., \& Khangulyan, D. 2011, PASJ, 63, 1023

Bosch-Ramon, V., Motch, C., Ribó, M., et al. 2007, A\&A, 473, 545

Bosch-Ramon, V., Khangulyan, D., \& Aharonian, F. A. 2008, A\&A, 482, 397

Bosch-Ramon, V., Barkov, M. V., Khangulyan, D., \& Perucho, M. 2012, A\&A, 544, A59

Bosch-Ramon, V., Barkov, M. V., \& Perucho, M. 2015, A\&A, 577, A89

Casares, J., Ribó, M., Ribas, I., et al. 2005, MNRAS, 364, 899

Cerutti, B., Malzac, J., Dubus, G., \& Henri, G. 2010, A\&A, 519, A81

Collmar, W., \& Zhang, S. 2014, A\&A, 565, A38

Corbet, R. H. D., Chomiuk, L., Coe, M. J., et al. 2016, ApJ, 829, 105

Corbet, R. H. D., Chomiuk, L., Coe, M. J., et al. 2019, ApJ, 884, 93

de la Cita, V. M., Bosch-Ramon, V., Paredes-Fortuny, X., Khangulyan, D., \& Perucho, M. 2016, A\&A, 591, A15

del Palacio, S., Bosch-Ramon, V., \& Romero, G. E. 2015, A\&A, 575, A112

Derishev, E. V., \& Aharonian, F. A. 2012, in American Institute of Physics Conference Series, eds. F. A. Aharonian, W. Hofmann, \& F. M. Rieger, Am. Inst. Phys. Conf. Ser., 1505, 402

Dubus, G. 2006, A\&A, 456, 801

Dubus, G. 2013, A\&ARv., 21, 64

Dubus, G., Lamberts, A., \& Fromang, S. 2015, A\&A, 581, A27

Dubus, G., Guillard, N., Petrucci, P.-O., \& Martin, P. 2017, A\&A, 608, A59

Eger, P., Laffon, H., Bordas, P., et al. 2016, MNRAS, 457, 1753

Eichler, D., \& Usov, V. 1993, ApJ, 402, 271

Fermi LAT Collaboration (Abdo, A. A., et al.) 2009, ApJ, 706, L56

Fermi LAT Collaboration (Ackermann, M., et al.) 2012, Science, 335, 189

Gaia Collaboration (Brown, A. G. A., et al.) 2018, A\&A, 616, A1

Gould, R. J., \& Schréder, G. P. 1967, Phys. Rev., 155, 1408

Hadasch, D., Torres, D. F., Tanaka, T., et al. 2012, ApJ, 749, 54

Hess, C., Abramowski, A., et al. 2015, MNRAS, 446, 1163

Hinton, J. A., Skilton, J. L., Funk, S., et al. 2009, ApJ, 690, L101

Khangulyan, D., Hnatic, S., Aharonian, F., \& Bogovalov, S. 2007, MNRAS, 380, 320

Khangulyan, D., Aharonian, F. A., \& Kelner, S. R. 2014, ApJ, 783, 100

Khangulyan, D., Aharonian, F., Romoli, C., \& Taylor, A. 2020, ArXiv e-prints [arXiv:2003.00927]

Leahy, D. A. 2004, A\&A, 413, 1019

Luri, X., Brown, A. G. A., Sarro, L. M., et al. 2018, A\&A, 616, A9

Maraschi, L., \& Treves, A. 1981, MNRAS, 194, 1P

Moldón, J., Ribó, M., \& Paredes, J. M. 2012, A\&A, 548, A103

Molina, E., \& Bosch-Ramon, V. 2018, A\&A, 618, A146

Molina, E., del Palacio, S., \& Bosch-Ramon, V. 2019, A\&A, 629, A129

Muijres, L. E., de Koter, A., Vink, J. S., et al. 2011, A\&A, 526, A32

Muijres, L. E., Vink, J. S., de Koter, A., Müller, P. E., \& Langer, N. 2012, A\&A, 537, A37

Pacholczyk, A. G. 1970, Radio Astrophysics. Nonthermal Processes in Galactic and Extragalactic Sources (W. H. Freeman \& Company)

Paredes, J. M., \& Bordas, P. 2019, ArXiv e-prints [arXiv:1901.03624]

Paredes, J. M., Martí, J., Ribó, M., \& Massi, M. 2000, Science, 288, 2340

Pauldrach, A., Puls, J., \& Kudritzki, R. P. 1986, A\&A, 164, 86

Rybicki, G. B., \& Lightman, A. P. 1986, Radiative Processes in Astrophysics (Wiley-VCH)

Sarty, G. E., Szalai, T., Kiss, L. L., et al. 2011, MNRAS, 411, 1293

Sierpowska-Bartosik, A., \& Torres, D. F. 2007, ApJ, 671, L145

Szostek, A., \& Dubus, G. 2011, MNRAS, 411, 193

Takahashi, T., Kishishita, T., Uchiyama, Y., et al. 2009, ApJ, 697, 592

Takata, J., Leung, G. C. K., Tam, P. H. T., et al. 2014, ApJ, 790, 18

Tavani, M., Kniffen, D., Mattox, J. R., Paredes, J. M., \& Foster, R. S. 1998, ApJ, 497, L89

Zabalza, V., Bosch-Ramon, V., \& Paredes, J. M. 2011, ApJ, 743, 7

Zabalza, V., Bosch-Ramon, V., Aharonian, F., \& Khangulyan, D. 2013, A\&A, 551, A17

Zanin, R., Fernández-Barral, A., de Oña Wilhelmi, E., et al. 2016, A\&A, 596, A55

Zdziarski, A. A., Malyshev, D., Dubus, G., et al. 2018, MNRAS, 479, 4399 